EPJ Web of Conferences 37, 09011 (2012)

DOI: 10.1051/epjconf/20123709011

(C) Owned by the authors, published by EDP Sciences, 2012

\title{
Studies of the Three-Nucleon System Dynamics in the Deuteron-Proton Breakup Reaction
}

Izabela Ciepał $^{1, a}$, Barbara Kłos ${ }^{2}$, St. Kistryn ${ }^{1}$, E. Stephan ${ }^{2}$, A. Biegun ${ }^{3}$, K. Bodek ${ }^{1}$, A. Deltuva ${ }^{5}$, E. Epelbaum ${ }^{15}$, M. Eslami-Kalantari ${ }^{3,16}$, A. C. Fonseca ${ }^{5}$, J. Golak ${ }^{1}$, V. Jha ${ }^{6}$, N. KalantarNayestanaki $^{3}$, H. Kamada ${ }^{7}$, G. Khatri ${ }^{1}$, Da. Kirillov ${ }^{14}$, Di. Kirillov ${ }^{8}$, St. Kliczewski ${ }^{4}$, A. Kozela ${ }^{4}$, M. Kravcikova ${ }^{9}$, H. Machner ${ }^{14}$, A. Magiera ${ }^{1}$, G. Martinska ${ }^{10}$, J. Messchendorp ${ }^{3}$, A. Nogga ${ }^{14}$, W. Parol ${ }^{1}$, A. Ramazani-Moghaddam-Arani ${ }^{3}$, B. J. Roy ${ }^{6}$, H. Sakai ${ }^{12}$, K. Sekiguchi ${ }^{13}$, I. Sitnik ${ }^{8}$, R. Siudak ${ }^{4}$, R. Skibiński ${ }^{1}$, R. Sworst ${ }^{1}$, J. Urban $^{10}$, H. Witała ${ }^{1}$, A. Wrońska ${ }^{1}$, and J. Zejma ${ }^{1}$

1 Jagiellonian University, PL-30059 Kraków, Poland

2 University of Silesia, PL-40007 Katowice, Poland

3 Kernfysisch Versneller Instituut, NL-9747 AA Groningen, The Netherlands

4 Institute of Nuclear Physics PAN, PL-31342 Kraków, Poland

5 Centro de Física Nuclear da Universidade de Lisboa, P-1649-003 Lisboa, Portugal

6 BARC, Bombay-400 085, India

7 Department of Physics, Kyushu Institute of Technology, Kitakyushu 804-8550, Japan

8 JINR, Dubna RU-141980 Dubna, Russia

9 Technical University, SK-04101 Kosice, Slovakia

10 P. J. Safarik University, SK-04154 Kosice, Slovakia

11 Universität Bonn, D-53113 Bonn, Germany

12 University of Tokyo, Bunkyo, J-1130033, Tokyo, Japan

13 Tohoku University, Sendai, J-9808578, Japan

14 Forschungszentrum Jülich, Institut für Kernphysik, D-52425 Jülich, Germany

15 Institut für Theoretische Physik II, Ruhr-Universität Bochum, D-44780 Bochum, Germany

16 Faculty of Physics, Yazd University, Yazd, Iran

\begin{abstract}
Precise and large sets of cross sections for the ${ }^{1} H(d, p p) n$ and ${ }^{2} H(p, p p) n$ breakup reactions were measured at 130 deuteron and $190 \mathrm{MeV}$ proton beam energies with the use of detectors which covered almost the full phase space. The results are compared with various theoretical approaches which model the $3 \mathrm{~N}$ system dynamics. The calculations are based on different $2 \mathrm{~N}$ potentials which can be combined with models of the three-nucleon force (3NF). The differential cross sections appear to be a very sensitive tool for testing the interaction models and allow to search for various ingredients of the dynamics, not only the $3 \mathrm{NF}$ but also the Coulomb interaction and relativistic effects which are relatively new achievements of the theory.
\end{abstract}

\section{Introduction}

Nowadays, there exist many models of nucleon-nucleon (NN) interaction like Av18, CD Bonn or family of Nijmegen potentials. They are constructed using the meson exchange theory or phenomenology. To test thoroughly these models, environement with more than just two nucleons is needed. The most suitable one is the three nucleon ( $3 \mathrm{~N})$ system, which can be studied in details with the use of the deuteron breakup reaction. Properties of few-nucleon systems at not too high energies are determined mainly by pairwise NN interaction. However, there are experimental clues which indicate the existence

a e-mail: izabela.ciepal@uj.edu.pl

This is an Open Access article distributed under the terms of the Creative Commons Attribution License 2.0, which permits unrestricted use, distribution, and reproduction in any medium, provided the original work is properly cited. 
of additional dynamics, refered to as three nucleon force (3NF). Moreover, other pieces of the system dynamics also participate, like Coulomb force or relativistic component.

The theoretical predictions of observables are obtained by means of rigorous solution of Faddeev equations. Except realistic NN potentials and supplementing them 3NF models (TM99 3NF or Urbana IX $3 \mathrm{NF}$ ), the $3 \mathrm{~N}$ system dynamics can be described within the coupled-channels (CC) approach [5]. This method is based on explicit treatment of a single $\Delta$-isobar degrees of freedom, what generates also certain $3 \mathrm{NF}$ effects. A still alternative way comes from chiral perturbation theory (ChPT). The nonvanishing $3 \mathrm{NF}$ appars at the next-to-next-to-leading (NNLO) order [8,9], which is numerically fully developed. Within the $\mathrm{CC}$ formalism the Coulomb interaction was implemented into calculations for the first time [6]. Recently, a consistent theoretical treatment of phenomenological 3NF and Coulomb force has been achieved also for the Av18+UIX potential [7] what allows to investigate the role of both effects on the high level of accuracy. Moreover, the relativistic treatment of the breakup reaction in $3 \mathrm{~N}$ system was developed for calculations using the NN potential [10] and this approach has been also extended for calculations includnig the $3 \mathrm{NF}$ [11].

The extensive and precise data sets which were obtained in series of new generation experiments are used to distinguish between the effects of various origins and to test the corresponding predictions.

\section{Experiments}

New-generation experiments dedicated to investigations of the $p(d, p p) n$ and $d(p, p p) n$ breakup reactions in a large phase space region were performed with the use of the SALAD and BINA detectors at KVI [1-4] and the Germanium Wall (GeWall) setup at FZ-Jülich [13]. The deuteron and proton beams of energies 130 and $190 \mathrm{MeV}$ respectively were used and large set of the breakup differential cross sections were measured.

The SALAD detector consisted of a three-plane multi-wire proportional chamber (MWPC) and two layers of a segmented scintillator hodoscope: horizontal $\Delta E$ and vertical stopping $E$ detectors. The acceptance of the setup covered the region of the polar angles from $12^{0}$ to $40^{\circ}$ and the full range of the azimuthal angles. The liquid hydrogen target was placed inside the scattering chamber. BINA apparatus was constructed as an upgraded version of SALAD and possesed two main parts called Wall and Ball. Wall inherited most parts and features from SALAD, covering the same angular range and built of the same MWPC and modified $\Delta E$ and $E$ hodoscopes. The backward part is ball-shaped and consists of 149 phoswich detectors which cover polar angles between $40^{\circ}$ and $160^{\circ}$. The Ball plays two roles: of particle detector and scattering chamber. In the measurements of the $d-p$ and $p-d$ breakup reactions liquid targets $\mathrm{LD}_{2}$ and $\mathrm{LH}_{2}$ were used.

The GeWall setup at the Research Center in Jülich (FZJ) consisted of three high-purity semiconductor position sensitive germanium detectors. Two different types of the detectors were used: a thin transmission detector "Quirl" with an excellent spatial resolution used to determine the position and energy loss ( $\triangle E$ detector) of the passing charged particles, and two thick energy detectors E1 and E2 with excellent energy resolutions. The angular acceptance of the apparatus was $3^{0}-14^{0}$ for the polar and $2 \pi$ for the azimuthal angles.

\section{Results}

In order to search for subtle dynamical effects in few-body systems precise and systematic database is necessary, what makes the mesurements very demanding. Our new-generation experiments fulfilled these conditions and provided a very rich set of differential cross sections for the breakup reactions. The cross sections were obtained for about 80 [2] and 94 [12] kinematical configurations, defined by the polar angles of the two outgoing protons, $\theta_{1}, \theta_{2}$, and their relative azimuthal angle $\varphi_{12}$, for the two energies of 65 and $190 \mathrm{MeV} /$ nucleon respectively with the use of the SALAD and BINA apparatus. Moreover, additional set of the cross sections for 145 geometries were obtained with the GeWall detector [13]. The elastic scattering process was measured simultaneously in each experiment 


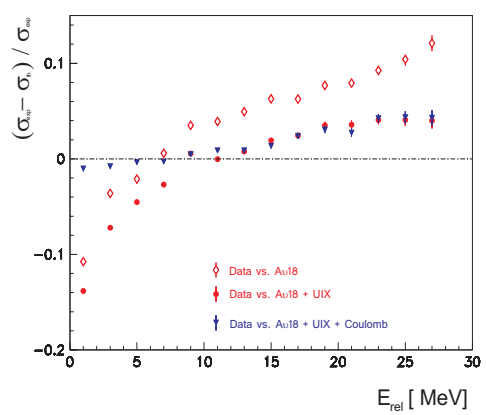

Fig. 1. Relative discrepancies between the experimental data and the theoretical predictions of the breakup cross sections as a function of the kinetic energy of the relative motion of the two breakup protons. The results for the pure Av18 NN potential (empty red diamonds), the Av18 combined with the Urbana IX $3 \mathrm{NF}$ ( full red dots) and same calculations but combined with Coulomb force (blue triangles) are presented.

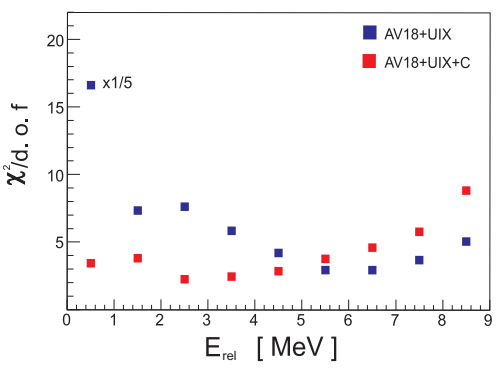

Fig. 2. Quality of description of the cross section data with the calculations based on Av18 NN potential combined with the Urbana IX $3 \mathrm{NF}$ (blue squares) and, in addition, with the Coulomb force included (red squares). Dependence of $\chi^{2} /$ d.o.f on the kinetic energy of the relative motion of the two breakup protons is shown. Point with a very large value of $\chi^{2} /$ d.o.f is scaled down by a factor of 15 , as indicated in the panel.

and the data were used for the breakup cross sections normalization. The obtained results allowed to trace different aspects of the system dynamics, 3NF effects, influence of the Coulomb force for the measured observables and the relativistic effects.

The cross section data obtained for $65 \mathrm{MeV} /$ nucleon at KVI were compared with the theoretical predictions and revealed both, the significant influence of the 3NF [1] and the Coulomb effects [2]. Importance of the $3 \mathrm{NF}$ for the proper data description is presented in Fig. 1. The relative difference of the experimental and theoretical cross sections, $\left(\sigma_{\text {exp }}-\sigma_{t h}\right) / \sigma_{\text {exp }}$, is presented as a function of the relative kinetic energy of the two breakup protons $E_{\text {rel }}$ for calculations based on Av18 potential and Av18 combining with the UIX 3NF model (Av18+UIX). The prediction containing the UIX 3NF significantly improves the data description in almost the whole range of $E_{r e l}$. However, at very small relative energy, there still exist quite large differencies between the data and the theory, and adding the UIX 3 NF makes the discrepancies even larger.

As it turned out the missing part of the dynamics is the Coulomb force and only calculations which take into account both dynamical components, the UIX 3NF and the Coulomb interaction, are able to remove the disagreements (see Fig. 1). Adding the electromagnetic force into the calculations does not essentially change the quality of the data description at large $E_{r e l}$, however at small $E_{r e l}$ values the observed discrepancies are almost totaly removed. As one can notice, there are still some discrepancies at large $E_{r e l}$ what can be interpreted as missing part of the dynamics, either the relativistic effects or unresolved problems in our understanding of the $3 \mathrm{NF}$ structure.

The Coulomb force were studied in more details in dedicated experiment at FZJ [13], focused on a very narrow part of the phase space. Dependencies of $\chi^{2}$ per degree of freedom (d.o.f.) as a function of the $E_{r e l}$ were studied, see Fig. 2. In the case of very small $E_{r e l}$ the Coulomb effects are extremaly high. It has been proven that only the predictions containing the Coulomb component are able to reproduce the data in a correct way.

The data measured at $190 \mathrm{MeV} /$ nucleon [12] reveal significant discrepancies in some geometrical configurations when comparing to the theoretical calculactions includning the $3 \mathrm{NF}$ potentials. Possible missing ingredients which could contribute are the relativistic effects. The relativistic calculations improve the data description only in certain parts of the phase space see Fig. 3.

\section{Summary and outlook}

Precise and systematic studies of the breakup reaction in a large part of the phase space are very important for understanding of the interaction between nucleons in few-nucleon systems. Currently 


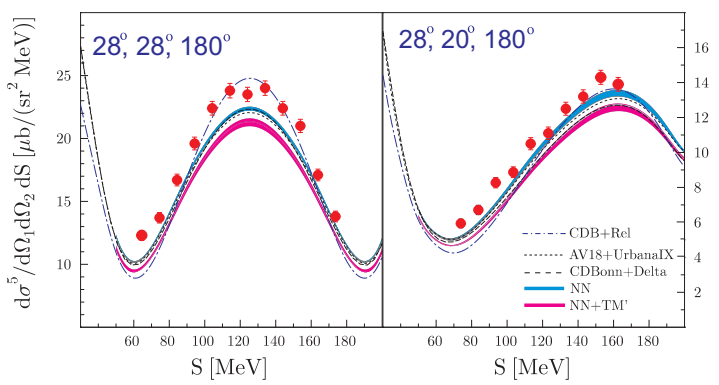

Fig. 3. Differential cross sections for the d(p, pp)n breakup reaction at $E_{p}=190 \mathrm{MeV}$ at two different kinematical configurations (specified in the panels) in which significant relativistic effects are observed. Experimental data are compared with results of different theoretical calculations described in the legend.

available theoretical approaches which try to model the interaction need very precise and large experimental database to be verified and further developed. Within these predictions different pieces of the dynamics can be studied separately and also their mutual interplay can be investigated. The data were obtained at several beam energies and in general confirmed the modern calculations, however there are still some problems with our understanding of the current models of the $3 \mathrm{NF}$. Moreover, there is still strong need to have possibly complete theoretical treatments including all ingredients of the $3 \mathrm{~N}$ system dynamics (3NF, Coulomb interaction, relativistic effects).

New experiments to study $3 \mathrm{~N}$ system dynamics are planned, including also investigations of the three-body system in the four-body environment. The future studies of the $3 \mathrm{~N}$ system dynamics in the deuteron breakup reaction with the use of the WASA detector at FZJ are focused on investigation of the relativistic effects and the role of the $3 \mathrm{NF}$ at higher energies. New scientific program concentrated on investigation of the few-nucleon systems dynamics at not too high energies was proposed to be carried out with the use of the BINA detector at the Cyclotron Center Bronowice in Cracow.

\section{Acknowledgements}

This work was partially supplemented by the Polish 2008-2010 science founds as a research Project No. N N202 174635 and by the Polish 2009-2011 science founds as a research Project No. N N202 034836. This publication is also supported by funding from the Jagiellonian University within the SET project. The project is co-financed by the European Union.

\section{References}

1. St. Kistryn et al., Phys. Rev. C 72, 044006 (2005).

2. St. Kistryn et al., Phys. Lett. B 641, 23-27 (2006).

3. E. Stephan et al., Phys. Rev. C 76, 057001 (2007).

4. E. Stephan et al., Phys. Rev. C 82, 014003 (2010).

5. A. Deltuva, A. Machleidt, P.U. Sauer, Phys. Rev. C 68, 024005 (2003).

6. A. Deltuva, A. C. Fonseca, P.U. Sauer, Phys. Rev. C 73, 057001 (2006).

7. A. Deltuva, Phys. Rev. C 80, 064002 (2009).

8. D. R. Entem, R. Machleidt, Phys. Lett. B 524, 93-98 (2002).

9. E. Epelbaum, Prog. Part. Nucl. Phys. 57, 645-741 (2006).

10. R. Skibiński, H. Witała, J. Golak, Eur. Phys. Jour. A 30, 369 (2006).

11. H. Witała et al., Phys. Rev. C 83, 044001 (2011).

12. N. Kalantar-Nayestanaki et al., Rep. Prog. Phys. 75, 016301 (2012).

13. E. Stephan et al., Int. Jour. Mod. Phys. A 24, 515-520 (2009). 